\title{
Situación actual de la gestión integrada de los residuos sólidos en el municipio de Cobán, Alta Verapaz, Guatemala, C. A.
}

\author{
Gustavo Adolfo García Macz \\ Candidato a Doctor del Programa DOCINADE -UNED- \\ Licenciado en Ciencias Agrícolas \\ M. Sc. en Gerencia para el Desarrollo Sostenible \\ ingusma@gmail.com \\ Profesor Titular \\ Centro Universitario del Norte \\ Universidad de San Carlos de Guatemala \\ Gesly Aníbal Bonilla Landaverry \\ Ingeniero Agrónomo, Maestro en Ciencias en Gestión Ambiental y \\ Doctor en Ciencias Políticas y Sociología \\ gesly77@hotmail.com \\ Centro Universitario de Sur Oriente \\ Universidad de San Carlos de Guatemala
}

Fecha de recepción: 15/11/2015 Fecha de aceptación: 07/03/2016

\begin{abstract}
Resumen
Se realizó en la cabecera municipal de Cobán, del departamento de Alta Verapaz, Guatemala, un estudio con la finalidad de establecer la situación actual de la Gestión Integral de Residuos Sólidos -GIRS- en esa localidad, por lo que se hizo un diagnóstico que proporcionó los factores básicos que determinan el estado del manejo, disposición y aprovechamiento de los residuos sólidos en Cobán. La investigación se sustentó en aspectos sociales, económicos, institucionales y ambientales para que con los resultados obtenidos se pueda fomentar el mejoramiento de la estructura administrativa municipal que se encargará de la gestión integrada de los residuos sólidos. Con los resultados que se presentan en este artículo se pretende dar a conocer a las autoridades municipales acerca de la necesidad de implementar métodos alternativos del manejo de la basura pues de lo contrario se continuará con los daños sociales e impactos ambientales que van en detrimento de la población en Cobán y del sistema ambiental en general.
\end{abstract}

\section{Palabras clave}

Gestión integral de residuos sólidos, impacto ambiental, contaminación ambiental, gestión ambiental

\begin{abstract}
It was held in the municipal head of Cobán, department of Alta Verapaz, Guatemala, a study in order to establish the current status of Integrated Solid Waste Management -GIRS- in that locality, so that was a diagnosis that provided the basic factors that determine the status of management, disposal and utilization of solid waste in Cobán. The research is based on social, economic, institutional and environmental aspects for the results obtained can encourage the improvement of the municipal administrative structure to be responsible for the integrated management of solid waste. With the results presented in this article is intended to inform local authorities about the need to implement alternative methods of waste management, otherwise it will continue with the social damage and environmental impacts that are detrimental to the Coban population and environmental system in general.
\end{abstract}

\section{Keywords}

Integral management of solid waste, environmental impact, environmental pollution, environmental management 


\section{Introducción}

En la mayoría de las municipalidades de Guatemala se ha mostrado débil gestión ambiental, lo cual no ha contribuido efectivamente con la protección de los recursos naturales y de la biodiversidad, al control de la contaminación, así como al debido ordenamiento territorial; las consecuencias son la disminución de la cobertura boscosa, vulnerabilidad socio ambiental, alteración a los ciclos hidrológicos, y daños socioeconómicos y ambientales en general (IARNA, 2004; PNUD, 2005).

Uno de los principales problemas ambientales que afronta la mayoría de las zonas urbanas de Guatemala son los residuos sólidos y parece ser que en el municipio de Cobán, Alta Verapaz, la gestión integrada de los residuos sólidos -GIRS- no se lleva a cabo con eficiencia, ya que no existe obligatoriedad en los pobladores, generada por las autoridades municipales para que se contraten los servicios de las empresas recolectoras $y$, no se hace un manejo integrado de residuos sólidos, ni en los hogares, ni por parte de las empresas recolectoras, las cuales solamente depositan la carga en el basurero municipal.

No obstante, teniendo una buena gestión de los residuos sólidos de forma integral, se podría propiciar una mejora en la calidad de vida de la población pues la Gestión Integral de Residuos Sólidos tiene como meta contribuir de manera armónica al manejo de los problemas ambientales y de la salud pública, ya que los mismos habitantes de un poblado pueden hacer un reuso y reciclaje de ciertos componentes de los residuos sólidos (AMSA, 2004).

En Cobán se encuentra un basurero municipal donde las personas recolectan materiales del contenido vertido, ya que esto representa un medio de subsistencia, pero el resto de los residuos sólidos, tanto orgánicos como inorgánicos vuelve a quedar mezclado, sin tratamiento. Con el correr del tiempo y en época de verano se originan incendios que producen contaminación y generan altas concentraciones de $\mathrm{CO}_{2}$ y otras emanaciones como gas metano, etano y propano, que generan material altamente combustible que a la vez de provocar polución generan peligro para la población cercana. Durante la carga y transporte de los desechos, los envases se rompen al manejarlos, además de que algunas bolsas se caen y quedan en la ruta, por lo que también los recolectores se constituyen en contaminadores de las vías de acceso.

El presente trabajo muestra el estudio que se realizó en la cabecera municipal de Cobán, en el departamento de Alta Verapaz, con la finalidad de establecer la situación actual de la Gestión Integral de los Residuos Sólidos -GIRS-, por lo que se inició con un diagnóstico que proporcionó los factores básicos que determinan el manejo de los residuos sólidos en Cobán. Las variables estudiadas fueron el manejo de los residuos sólidos -RS- en los hogares, tren de limpieza municipal, depósitos clandestinos de RS, RS en las calles, la opinión de la población acerca del estado actual y sugerencias de las personas y otras que se identifiquen en el proceso.

Con los resultados obtenidos se pretende dar a conocer a las autoridades municipales acerca de la necesidad de implementar métodos alternativos al manejo de la basura porque puede provocar grandes daños a la salud de los seres humanos, contamina el ambiente y reduce la belleza del entorno. Es conveniente retomar los valores y principios ambientales de la población a través de la educación para que mejoren su conducta frente al ambiente y el daño que causa la basura. La idea básica es orientar a los usuarios a separar los materiales recuperables que son retirados en forma especial y conducidos a industrias que los utilizan como materia prima para la elaboración de sus productos, lográndose obtener beneficios económicos. 


\section{Marco teórico}

\subsection{Residuos sólidos}

Los residuos sólidos -RS- se definen como todos los tipos de materiales resultantes como empaques, envases, cubiertas, protectores de enseres, ropa inutilizable, restos de alimentos, materiales para higiene y limpieza y otros provenientes de los servicios prestados y recibidos por el humano en el hogar, comercio, industria, hospitales, en fin, todos aquellos desechos resultantes de la actividad humana (Zorrilla, 2005).

Según Mijangos (2002), los desechos sólidos comprenden todos los residuos que provienen de actividades humanas y de producción animal, que normalmente son sólidos y desechados como inútiles y superfluos y se pueden clasificar como sólidos orgánicos, recuperables y no recuperables. Los sólidos orgánicos constituyen casi todos los casos hasta el 50\% del total de los residuos domésticos, o bien toda aquella materia que puede descomponerse en otra más sencilla; normalmente con restos de comida y hojarasca. Los desechos sólidos recuperables son aquellos que una vez seleccionados pueden reutilizarse $y / o$ venderse a diferentes industrias, tales como: vidrio, papel, cartón, plástico, trapos, huesos, maderas, metales, entre otros. Por su parte, los desechos sólidos no recuperables pueden ser peligrosos, nocivos o inertes; tales como; los desperdicios provenientes de hospitales.

De hecho, todos los hospitales están obligados a cumplir la normativa para ser eliminados (Código de Salud, Dto 90-97). Sin embargo, de acuerdo a Recinos (2014), hay personas particulares que se dedican a realizar tareas en casa de administración de medicamentos hipodérmicos, depositan jeringas, bisturíes y otros materiales peligrosos en las bolsas de residuos sólidos comunes. Dentro de los residuos sólidos municipales no se incluyen "desechos de construcción y vehículos automotores y no motores, lodo, ceniza de combustión y basu- ra de proceso industrial y artesanal (Van de Kludndert y Anschultz, 2001). En Cobán, por ejemplo, varios talleres de carpintería, zapatería, sastrería, etc., incorporan sus desechos en la basura común, incluyendo los de jardinería.

El volumen y tipo de basura sólida generada en zonas urbanas está determinado por las características y tamaño del centro urbano. En países industrializados los desechos sólidos representan una mínima proporción de todos los residuos sólidos, no obstante en ciudades como Cobán, los RS forman la mayor parte. Cabe indicar que en los países latinoamericanos, son pocos los datos reportados, tal es el caso de Chile, en donde diversos estudios indican que la producción de desechos sólidos per cápita oscila entre 4,2 y 4,8 kg/día (Extraño, 2002).

En los grupos sociales, por homogéneos que sean, siempre existen costumbres heredadas, que determinan el patrón de conducta observado; en Cobán los pobladores están formados por diversas etnias, en su mayoría Q'eqchí (PNUD, 1999) lo que hace que se den diversos métodos de tratamiento de los residuos sólidos: incineración, dispersión en el suelo como abono, enterrada y otras formas dadas en las residencias y por eso se afirma que "desde muchos puntos de vista, los residuos sólidos constituyen el flujo de residuos más importante porque proviene de todos nosotros, reflexionando sobre nuestro estilo de vida, es necesario analizar nuestro consumo de recursos y opciones de recuperación de los mismos".

La mayoría de municipios a través de sus gobiernos deberían colocar a la gestión integrada de residuos sólidos como la solución primordial al problema; sin embargo -si lo hacen- la constante es el traslado de los RS a un botadero de residuos sin ningún tratamiento previo o el destino final del problema es un relleno sanitario. En Cobán solamente existe un basurero municipal, al cual llega la mayoría de RS ya que no todas las personas pagan el servicio de recolección. 


\subsection{Efectos de los desechos sólidos al ambiente}

Los aspectos ambientales más prominentes de los residuos sólidos son: el deterioro estético del paisaje natural, contaminación del agua, suelo y aire (MARN, 2001). Según Mijangos (2002), los efectos contaminantes de los desechos sólidos en las aguas, ocurre tanto en las aguas superficiales como en las subterráneas, por el vertido directo de la basura a los ríos y quebradas y por la mala disposición de líquido percolado (lixiviado) producto de los botaderos a cielo abierto.

\subsection{Residuos sólidos como parte de la gestión ambiental y el desarrollo}

Parte de la gestión ambiental es la creación de leyes y políticas públicas de preferencia municipales que vayan en beneficio de la sociedad; en Guatemala se ha puesto atención al asunto con la creación de las leyes generales y sus reglamentos: Ley de Protección y Mejoramiento del Medio Ambiente (Decreto 68-86), Ley de Áreas Protegidas (Decreto 64-86), la cual mantiene aproximadamente el $40 \%$ de la cobertura boscosa del país en las áreas protegidas; también ha ratificado los tratados internacionales. Lo anterior confirma que el componente ambiental es visto como una oportunidad que brinda bienes y servicios ambientales a la sociedad guatemalteca, tales como disponibilidad de agua, productos maderables, energéticos, seguridad alimentaria y medicinas, entre otros, con justa valoración de la naturaleza por parte de la ciudadanía (MARN, 2001).

En respuesta a este tipo de demandas y preocupaciones emanadas por diferentes actores y sectores de la sociedad, el Ministerio de Ambiente y Recursos Naturales -MARN- de Guatemala, presenta el documento de política marco que fue elaborado en coordinación y apoyo de un grupo asesor conformado por ex- pertos relacionados con la Gestión Ambiental y de políticas públicas con una propuesta inicial (FIPA-USAID, 2 001, PAF-Maya, 2 002), luego en una segunda consulta se sugiere el cambio y actualización del planteamiento del 2002, el cual quedaría acondicionado a las circunstancias y requerimientos técnicos y legales de la época (UPIE-MAGA, MARN, CBM, DOM/GTZ, CATIE, IDEADS, ASOREMA, 2 003) y como producto se define la política ambiental marco que contempla dos grandes áreas: la primera, el patrimonio natural y la segunda la calidad ambiental, dentro de la cual se considera la gestión de los desechos sólidos. Las áreas antes mencionadas están relacionadas con siete ejes de política y con tres ámbitos temáticos (institucional, económico-productivo y social).

Los principios de la política marco manifiestan la expresión de diferentes sectores y actores, así como de marcos políticos nacionales e internacionales (Política Marco de Gestión ambiental, MARN, 2009). Los mismos son los siguientes:

a) Desarrollo Sostenible

b) El Bien común priva sobre el Bien Privado

c) Participación Ciudadana y Corresponsabilidad Social

d) Protección a la Diversidad Biológica

e) Equidad social

f) Equidad de género

g) Respeto a la Interculturalidad y Multiculturalidad

h) Precautoriedad

i) Ética ambiental

j) Corresponsabilidad, coordinación y cooperación interinstitucional y sectorial 


\subsection{Fuentes y clasificación de los residuos sólidos}

\subsubsection{Fuentes}

El conocimiento de los orígenes y los tipos de residuos sólidos, así como los datos sobre la composición y las tasas de generación, es básico para el diseño y la operación de los elementos funcionales asociados con la gestión de residuos sólidos. El origen de los residuos sólidos en una comunidad está, en general, relacionado con el uso del suelo y su localización. El residuo se puede clasificar de varias formas, tanto por estado, origen o característica (Zorrilla, 2005).

Las iniciativas (Careaga, 1993), para prevenir la generación de residuos son una contribución muy importante a la estrategia de manejo integral de residuos sólidos, esto se debe a que reducen la cantidad de materiales desechados que requieren tratamiento. Más aún, el concepto de reducción ayuda a elevar la conciencia del público en el manejo de los residuos sólidos, aunque dicha reducción debe ser evaluada cuidadosamente para asegurar que tenga bases científicas, ya que decisiones arbitrarias basadas en información sin fundamento pueden resultar en la disminución de una parte del flujo de residuos a costa de un mayor uso de recursos.

En los países en donde ya existe una conciencia ambiental los fabricantes tienen incentivos económicos y ambientales para darle al consumidor productos de la manera más eficiente posible. La reducción debe hacerse caso por caso tomando en cuenta el ciclo de vida del producto en cuestión. De esta manera, se previene que los problemas sólo cambien de lugar, ya que una mejora aparente en una parte del ciclo de vida puede simplemente llevar a otros problemas posteriores.

Un manejo integral de residuos sólidos exitoso, requiere que los miembros de la sociedad que contribuyen a integrar el flujo de residuos asuman sus responsabilidades. Productores de materias primas, fabricantes, distribuidores, comerciantes, consumidores y autoridades deben responsabilizarse por los residuos que generan. Una manera efectiva de promover la minimización de residuos experimentada en otros países, ha sido cobrar al generador de éstos conforme a la cantidad producida; ésta es una aplicación del principio "el que contamina paga" y forma parte de una estrategia de responsabilidad compartida.

\subsubsection{Clasificación por estado}

Un residuo es definido por estado según el estado físico en que se encuentre. Existen por lo tanto tres tipos de residuos desde este punto de vista sólidos, líquidos y gaseosos, es importante notar que el alcance real de esta clasificación puede fijarse en términos puramente descriptivos o, como es realizado en la práctica, según la forma de manejo asociado.

\subsubsection{Clasificación por origen}

Se puede definir el residuo por la actividad que lo origine, esencialmente es una clasificación sectorial. Esta definición no tiene en la práctica límites en cuanto al nivel de detalle en que se puede llegar a ella.

\subsubsection{Clasificación por tipo de manejo}

Se puede clasificar un residuo por presentar algunas características asociadas a manejo que debe ser realizado (Recinos, 2014). Desde este punto de vista se pueden definir tres grandes grupos:

a) Residuo peligroso: Son residuos que por su naturaleza son inherentemente peligrosos de manejar y/o disponer y pueden causar muerte, enfermedad; o que son peligrosos para la salud o el medio ambiente cuando son manejados en forma inapropiada. 
b) Residuo inerte: Residuo estable en el tiempo, el cual no producirá efectos ambientales apreciables al interactuar en el medio ambiente.

c) Residuo no peligroso: Ninguno de los anteriores.

\subsection{Tipos de residuos más importantes}

\subsubsection{Residuos municipales}

La generación de residuos municipales varía en función de factores culturales asociados a los niveles de ingreso, hábitos de consumo, desarrollo tecnológico y estándares de calidad de vida de la población. El creciente desarrollo de la economía chilena ha traído consigo un considerable aumento en la generación de estos residuos. Los sectores de más altos ingresos generan mayores volúmenes per cápita de los residuos, y estos tienen un mayor valor incorporado que los provenientes de sectores más pobres de la población (Zorrilla, 2005).

\subsubsection{Residuos industriales}

La cantidad de residuos que genera una industria es función de la tecnología del proceso productivo, calidad de las materias primas 0 productos intermedios, propiedades físicas y químicas de las materias auxiliares empleadas, combustibles utilizados y los envases y embalajes del proceso.

\subsubsection{Residuos mineros}

Los residuos mineros incluyen los materiales que son removidos para ganar acceso a los minerales y todos los residuos provenientes de los procesos mineros. Actualmente la industria del cobre se encuentra empeñada en la implementación de un manejo apropiado de estos residuos, por lo cual se espera en un futuro próximo contar con estadísticas apropiadas (Zorrilla, 2005).

\subsubsection{Residuos hospitalarios}

En la actualidad el manejo de los residuos hospitalarios no es el más apropiado, al no existir seguimiento y aplicación a los reglamentos. El manejo de estos residuos es realizado a nivel de generador y no bajo un sistema descentralizado. A nivel de hospital los residuos son generalmente esterilizados (Recinos, 2014).

\subsection{Composición de los residuos sólidos}

Usualmente los valores de composición de residuos sólidos municipales o domésticos se describen en términos de porcentaje en masa, también en base húmeda y contenidos como materia orgánica, papeles y cartones, escombros, plásticos, textiles, metales, vidrios, huesos, etc. La utilidad de conocer la composición de residuos sirve para una serie de fines, entre los que se pueden destacar estudios de factibilidad de reciclaje, factibilidad de tratamiento, investigación, identificación de residuos, estudio de políticas de gestión de manejo.

\subsection{Propiedades físicas de los residuos sólidos}

\subsubsection{Humedad}

Es una característica importante para los procesos a que puede ser sometida la basura. Se determina generalmente de la siguiente forma: Tomar una muestra representativa, de 1 a $2 \mathrm{Kg}$, se calienta a $80{ }^{\circ} \mathrm{C}$ durante 24 horas, se pesa y se expresa en base seca o húmeda. La Humedad es igual a (peso inicial-peso final)*100/ peso inicial

\subsubsection{Densidad}

La densidad de los sólidos rellenados depende de su constitución y humedad, porque este valor se debe medir para tener un valor más real. Se deben distinguir valores en distintas etapas del manejo. 


\subsubsection{Capacidad de campo}

Es la cantidad total de humedad que puede ser retenida por una muestra de residuo sometida a la acción de la gravedad, es de importancia crítica para determinar la formación de la lixiviación en los vertederos. Lixiviación es el exceso de agua sobre la capacidad de campo.

\subsubsection{Permeabilidad de los residuos sólidos compactados}

La conductividad hidrológica de los residuos sólidos compactados es una propiedad física importante que, en gran parte, gobierna el movimiento de líquidos y gases dentro de un vertedero.

\subsubsection{Esfuerzo cortante}

Los residuos sólidos tienen un esfuerzo cortante que se sabe que es máximo justo después de la compactación y disminuye con el paso del tiempo llegando a cero tras varios años de estancia en el vertedero.

\subsection{Otras propiedades de los residuos sólidos}

Según Zorrilla (2005), la composición química de los componentes que conforman los residuos sólidos es importante para evaluar las opciones de procesamiento y recuperación. Si los residuos sólidos van a utilizarse como combustibles, las propiedades más importantes que es preciso conocer son:

a) Análisis físico. El análisis físico incluye para los componentes combustibles de los residuos sólidos los siguientes ensayos:

- Humedad (pérdida de humedad cuando se calienta a $105{ }^{\circ} \mathrm{C}$ durante una hora).

- Materia volátil combustible, (pérdida de peso adicional con la ignición a $950{ }^{\circ} \mathrm{C}$ en un crisol cubierto).
- Carbón fijo, (rechazo combustible dejado después de retirar la materia volátil).

- Ceniza, (peso del rechazo después de la incineración en un crisol abierto).

b) Punto de fusión de la ceniza. Este se define como la temperatura en la que la ceniza resultante de la incineración de residuos se transforma en sólido (escoria) por la fusión y aglomeración. Las temperaturas típicas de fusión para la formación de escoria de residuos sólidos oscilan entre 1100 y $1200^{\circ} \mathrm{C}$.

\subsection{Propiedades biológicas}

Las propiedades biológicas son importantes para la tecnología de la digestión aerobia/ anaerobia en la transformación de residuos en energía y en productos finales beneficiosos. El proceso anaerobio implica la descomposición biológica de residuos alimenticios con productos finales de metano, dióxido de carbono y otros. Algunos componentes orgánicos de residuos sólidos no son deseables en la conversión biológica, como plásticos, gomas, pieles y madera. Los fragmentos importantes en la transformación biológica son las grasas, proteínas, la lignina, celulosa, hemicelulosa, lignocelulosa y los constituyentes solubles (Zorrilla, 2005).

\subsection{Tasas de generación y recogida de los residuos sólidos}

\subsubsection{Tasas de generación}

a) Producción per cápita -PPC-. La producción de residuos sólidos domésticos es una variable que depende básicamente del tamaño de la población y de sus características socioeconómicas. Una variable necesaria para dimensionar el sitio de disposición final es la llamada Producción per cápita -PPC-. Este parámetro asocia el tamaño de la población, la cantidad de residuos y el tiempo; siendo la unidad de 
expresión el kilogramo por habitante por día (Kg/hab/día).

b) Estimación teórica de producción per cápita -PPC-. La PPC es un parámetro que evoluciona en la medida que los elementos que la definen varían. En términos gruesos, la PPC varía de una población a otra, de acuerdo principalmente a su grado de urbanización, su densidad poblacional y su nivel de consumo o nivel socioeconómico. Otros elementos, como los periodos estacionales y las actividades predominantes también afectan la PPC.

c) Recogida de los residuos sólidos. Los residuos recolectados incluyen los residuos no seleccionados (en comunidades sin programa de reciclaje) y residuos no seleccionados y residuos seleccionados en ori- gen (en comunidades con programa de reciclaje). La diferencia entre la cantidad de residuos sólidos domésticos y comerciales y la cantidad de residuos recolectados para su procesamiento y / o vertido variará normalmente desde el 4 al 15\%. Las diferencias se pueden justificar por la cantidad del material 1) fermentado, 2) quemado en chimeneas domésticas, 3) arrojado en las alcantarillas, 4) donado a agencias de caridad, 5) vertido a mercadillos, 6) entregado a estaciones de recolección selectiva y centros de reciclaje 7) reciclado directamente. En general la diferencia porcentual entre la cantidad generada y la recolectada será más pequeña (del 4 al 6\%) para piso que para casas individuales con espacio adecuado para el compostaje doméstico en el jardín (del 8 al 15\%).

\section{Ubicación del área de estudio}

Figura 1

Mapa de ubicación del municipio de Cobán, A.V. y su área urbana en el contexto de Centro América

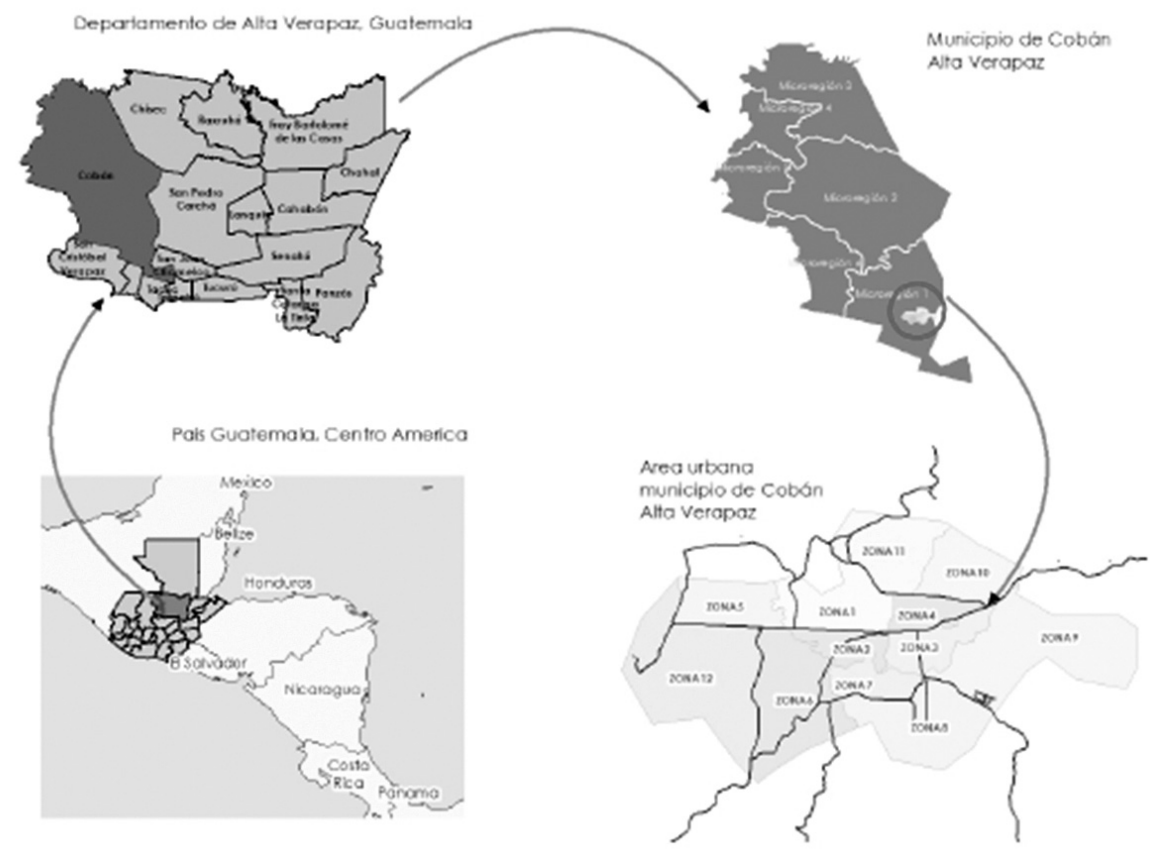

Fuente: Base Cartográfica del IGN e INE, Municipalidad de Cobán, A.V. y datos tomados en campo Elaboración: MSc. Gustavo García 
Programa de Doctorado: Ciencias Naturales para el Desarrollo con énfasis en gestión y cultura ambiental UNED, ITCR, UNA.

Figura 2

Mapa de ubicación del botadero municipal en el área urbana de Cobán, Alta Verapaz

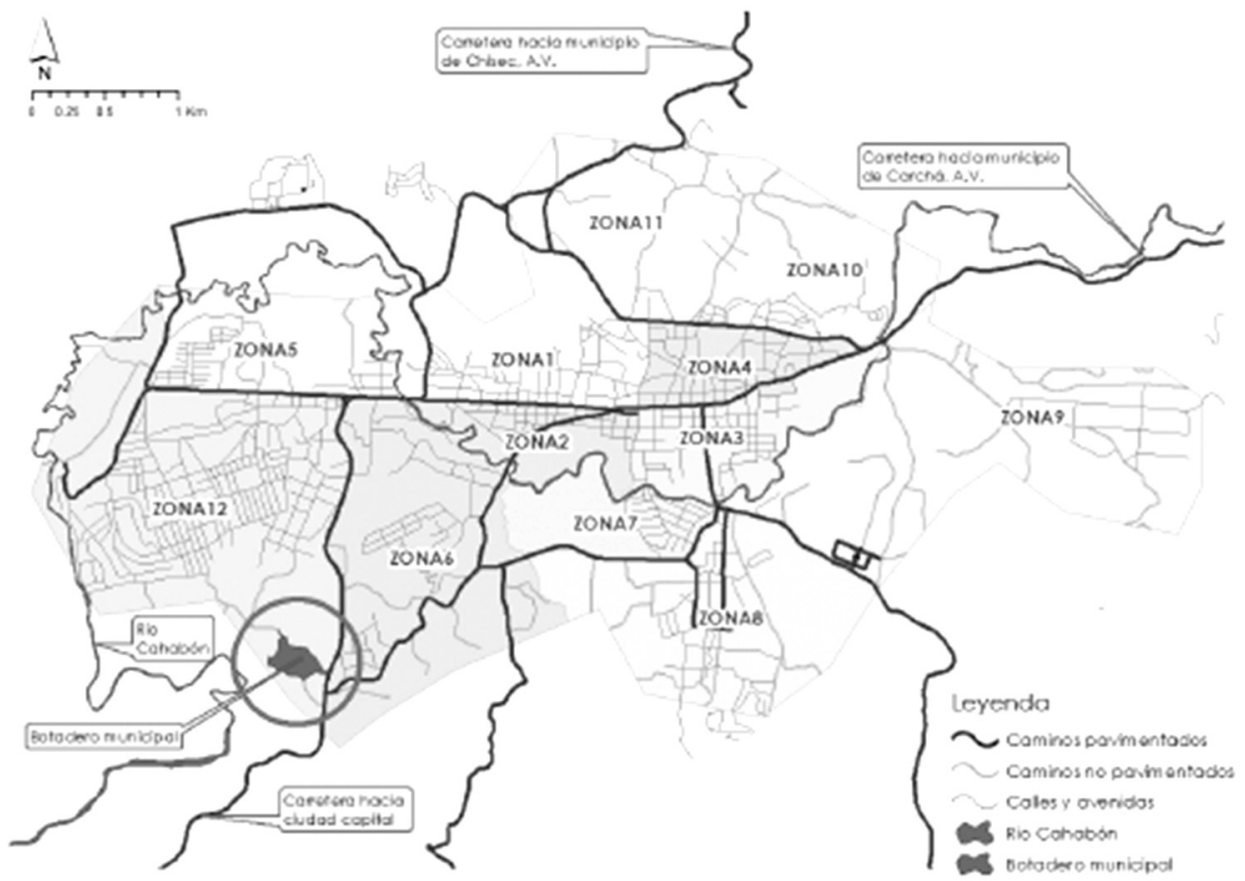

Fuente: Base Cartográfica del IGN e INE, Municipalidad de Cobán, A.V. y datos tomados en campo Elaboración: MSc. Gustavo García

\section{Resultados y discusión}

\subsection{Composición másica y proporción financiera de RS vertidos en el basurero}

Se realizó un análisis de la composición en materia de los RS que son producidos y vertidos en el basurero denominado Sachamach. El pa- pel y cartón representa un $15 \%$ en su proporción másica, no obstante su proporción financiera alcanza un 33,30\%. En cuanto al plástico, metales y vidrio constituyen el $41 \%$ del total, el resto está constituido por material orgánico que es biodegradable. Esto se presenta en la Tabla 1: 


\section{Tabla 1}

Composición global de los residuos sólidos en el vertedero de Sachamach, Cobán, Alta Verapaz, Guatemala

\begin{tabular}{|c|c|c|c|c|c|}
\hline \multicolumn{6}{|c|}{ Comercialización } \\
\hline Tipo de material & $\begin{array}{l}\text { Cantidad } \\
(\mathrm{Kg})\end{array}$ & $\begin{array}{l}\text { Proporción } \\
\text { másica (\%) }\end{array}$ & $\begin{array}{l}\text { Precio medio } \\
\text { (US\$/kg) }\end{array}$ & Total (US\$) & $\begin{array}{c}\text { Proporción } \\
\text { financiera (\%) }\end{array}$ \\
\hline \multicolumn{6}{|c|}{ Material No Biodegradable } \\
\hline Papel y cartón & $5.812,50$ & 15,00 & 0,200 & $1.162,50$ & 33,30 \\
\hline Plástico & $9.504,60$ & 14,00 & 0,180 & $1.710,83$ & 49,00 \\
\hline Metal & $1.255,50$ & 0,00 & 0,270 & 338,99 & 9,71 \\
\hline Vidrio & $1.116,00$ & 2,00 & 0,250 & 279,00 & 7,99 \\
\hline Subtotal & $17.919,00$ & 31,00 & 0,195 & $3.491,31$ & 100,00 \\
\hline \multicolumn{6}{|c|}{ Material Biodegradable } \\
\hline Orgánico & $43.704,00$ & & & & 100,00 \\
\hline
\end{tabular}

Fuente: Elaboración propia.

Los desechos orgánicos que se pueden separar y convertir en fertilizante orgánico son del orden del 20 a $30 \%$ del total biodegradable y se puede obtener un total de abono en proporción de 1:3, lo que indica que con 45 kilogramos de restos procesables se pueden obtener 15 kilogramos de abono, los cuales tienen un costo de US\$10,00. No obstante hay que tomar en cuenta que solamente el 0,0004\% (equivalente a una proporción de 24 personas) clasifica los residuos sólidos en el municipio y procesa los desechos orgánicos.

Con estos datos se puede inferir que la separación de los desechos biodegradables para elaborar compost puede generar ingresos y hacer una producción de plantas de jardín, pastos y otros cultivos de una forma amigable con el ambiente y que además, economiza a las personas el costo del fertilizante formulado.

La estrategia integral tiene varias ventajas:

- $\quad$ Proporciona un panorama global del proceso del manejo de los residuos. Esta visión es esencial para una planeación es- tratégica. Manejar el flujo de los residuos por separado es ineficiente.

- Desde un punto de vista ambiental todos los sistemas de manejo de residuos son parte de un mismo ecosistema global. Analizar la carga global del sistema al medio ambiente es la única estrategia racional, de otra manera reducir impactos ambientales en una parte del sistema, podría resultar en mayores impactos ambientales en otra parte del mismo.

- Desde un punto de vista económico, cada unidad individual en la cadena de manejo de residuos debe tener ganancias o al menos alcanzar un punto de equilibrio. Sin embargo, evaluando los límites del sistema en su totalidad, es posible determinar si opera eficientemente, si alcanza un punto de equilibrio económico o, mejor aún, si existen ganancias. Es solamente entonces cuando todas las partes del sistema pueden ser viables, asumiendo que los recursos se dividen apropiadamente en relación con los costos. Para alcanzar un sistema 
integral de manejo de residuos se requieren cambios significativos de la realidad actual. Es claro que nunca se alcanzará el sistema ideal, ya que siempre será posible reducir los impactos ambientales a través de un proceso de mejora continua.

- Para alcanzar un manejo de residuos sólidos, ambiental y económicamente sustentable, se requiere trabajar en un sistema diseñado para este propósito. Esto es clave ya que tratando de mejorar los sistemas actuales, agregando el reciclado o compostaje como "extras" al sistema, es probable que no funcione. Cuando se diseñe o rediseñe un sistema de manejo de residuos la estrategia debe considerar el sistema en su totalidad.

- Los diferentes componentes de un sistema están interconectados de tal manera que es necesario diseñar un sistema nue- vo en su totalidad, en vez de arreglar el viejo. Por ejemplo, la adición del reciclado a un sistema de manejo de residuos agrega el costo del sistema de reciclado al costo del sistema original de manejo de residuos. Una estrategia sistemática aseguraría que el costo del reciclado se mantuviera al mínimo, operando menos recolecciones para materiales secos 0 no-orgánicos, pues habría menos de estos residuos en virtud de que el material reciclable ya habría sido removido.

En cuanto a los residuos inorgánicos es necesario hacer notar que existe un alto porcentaje de plásticos y metales, los cuales por su naturaleza constituyen un gran porcentaje de elementos no biodegradables. Las cantidades y proporciones de los residuos sólidos inorgánicos que se depositan en el vertedero municipal se muestran en la Tabla 2:

\section{Tabla 2}

Residuos sólidos inorgánicos determinados en el vertedero municipal, del área urbana de Cobán, Alta Verapaz

\section{Resultado Global}

Comercialización

\begin{tabular}{lrrrrr}
\hline Tipo de material & Cantidad [kg] & $\begin{array}{c}\text { Proporción } \\
\text { másica [\%] }\end{array}$ & $\begin{array}{c}\text { Precio medio } \\
{[\text { US\$/kg] }}\end{array}$ & $\begin{array}{c}\text { Total } \\
{[\text { US } \$]}\end{array}$ & $\begin{array}{c}\text { Proporción } \\
\text { financiera [\%] }\end{array}$ \\
\hline Papel y cartón & $5,812.50$ & $15.00 \%$ & 0.200 & $1,162.50$ & $33.30 \%$ \\
\hline Plástico & $9,504.60$ & $14.00 \%$ & 0.180 & $1,710.83$ & $49.00 \%$ \\
Metales & $1,255.50$ & $0.00 \%$ & 0.270 & 338.99 & $9.71 \%$ \\
Vidrio & $1,116.00$ & $2.00 \%$ & 0.250 & 279.00 & $7.99 \%$ \\
\hline Total & $17,919.00$ & $31.00 \%$ & 0.195 & $3,419.31$ & $100.00 \%$ \\
\hline
\end{tabular}

Fuente: Elaboración propia.

El cuadro anterior, refleja el resumen de los residuos sólidos correspondientes al material inorgánico. Dentro de los plásticos se encontró Policloruro Vinil Carbono -PVC- en cantidades altas, lo cual es un indicador muy negativo en el sentido contaminante y la influencia de los valores, principios y actitudes, de la población que no percibe, el daño al entorno natural que le ocasiona la deposición de RS, sin tratamiento alguno. En ese sentido se cuantificó la cantidad de materiales derivados del plástico cuyos resultados se muestran en la Tabla 3: 


\section{Tabla 3}

\section{Residuos sólidos plásticos determinados}

\begin{tabular}{|c|c|c|c|c|}
\hline \multicolumn{5}{|c|}{ Comercialización } \\
\hline Tipo de material & Cantidad [kg] & $\begin{array}{l}\text { Proporción } \\
\text { másica [\%] }\end{array}$ & $\begin{array}{c}\text { Precio medio } \\
\text { [US\$/kg] }\end{array}$ & Total [US\$] \\
\hline 009 PET (1) & $5,134.48$ & $54.02 \%$ & 0.18 & 924.21 \\
\hline 010 Polietileno Alta Densidad PEAD (2) & 572.28 & $6.02 \%$ & 0.18 & 103.01 \\
\hline 011 Cloruro de Polivinilo PVC (3) & 664.45 & $6.99 \%$ & 0.18 & 119.60 \\
\hline 012 Polietileno Baja Densidad PEBD (4) & 756.49 & $7.96 \%$ & 0.18 & 136.17 \\
\hline 013 Polipropileno PP (5) & 478.23 & $5.03 \%$ & 0.18 & 86.08 \\
\hline 014 Poliestireno PS (6) & 474.95 & $5.00 \%$ & 0.18 & 85.49 \\
\hline 015 Otros plásticos (7) & $1,423.72$ & $14.98 \%$ & 0.18 & 256.27 \\
\hline Total & $9,504.60$ & $100.00 \%$ & 0.180 & $1,710.83$ \\
\hline
\end{tabular}

Fuente: Datos de campo

Con relación a los metales, el más hallado fue aluminio, cobre, hierro y acero, lo cual satura el relleno sanitario y dificulta el manejo de los residuos en el área estudiada. Los resultados de las cantidades de residuos sólidos metálicos encontrados en el vertedero municipal de Cobán se muestran en la Tabla 4:

Tabla 4

Residuos sólidos metálicos determinados

\begin{tabular}{|c|c|c|c|c|}
\hline \multicolumn{5}{|c|}{ Comercialización } \\
\hline Tipo de material & Cantidad [kg] & $\begin{array}{l}\text { Proporción másica } \\
{[\%]}\end{array}$ & $\begin{array}{l}\text { Precio medio } \\
\text { [US\$/kg] }\end{array}$ & Total [US\$] \\
\hline 016 - Aluminio (latas) & 377.56 & $30.07 \%$ & 0.27 & 101.94 \\
\hline 017 - Aluminio (bloque) & - & $0.00 \%$ & 0.27 & - \\
\hline 018 - Aluminio (perfil) & - & $0.00 \%$ & 0.27 & - \\
\hline 019 - Aluminio (olla) & 126.77 & $10.10 \%$ & 0.27 & 34.23 \\
\hline 020 - Aluminio marmitex & - & $0.00 \%$ & 0.27 & - \\
\hline 021 - Aluminio (diversos) & 62.76 & $5.00 \%$ & 0.27 & 16.95 \\
\hline 022 - Cobre forrado & 50.22 & $4.00 \%$ & 0.27 & 13.56 \\
\hline 023 - Cobre limpio & 37.12 & $2.96 \%$ & 0.27 & 10.02 \\
\hline 024 - Bronce & 81.89 & $6.52 \%$ & 0.27 & 22.11 \\
\hline 025 - Antimonio & - & $0.00 \%$ & 0.27 & - \\
\hline 026 - Metales/mixto & 98.44 & $7.84 \%$ & 0.27 & 26.58 \\
\hline
\end{tabular}

Continúa... 


\begin{tabular}{|c|c|c|c|c|}
\hline \multicolumn{5}{|c|}{ Comercialización } \\
\hline Tipo de material & Cantidad [kg] & $\begin{array}{c}\text { Proporción másica } \\
{[\%]}\end{array}$ & $\begin{array}{l}\text { Precio medio } \\
\text { [US\$/kg] }\end{array}$ & Total [US\$] \\
\hline $027-\operatorname{lnox}$ & - & $0.00 \%$ & 0.27 & - \\
\hline 028 - Chatarra de hierro & 285.88 & $22.77 \%$ & 0.27 & 77.19 \\
\hline 029 - Chatarra de computadora & 124.55 & $9.92 \%$ & 0.27 & 33.63 \\
\hline 030 - Diversos & 10.31 & $0.82 \%$ & 0.27 & 2.78 \\
\hline Total & $1,255.50$ & $100.00 \%$ & 0.270 & 338.99 \\
\hline
\end{tabular}

Fuente: Elaboración propia

Es importante señalar, que también se localizó mucho material a base de cadmio, litio y otros residuos provenientes de aparatos electrónicos, los cuales son de naturaleza radioactiva y que contaminan no sólo el recurso edáfico e hídrico sino también el atmosférico.
Para sintetizar la proporción másica de los materiales y/o residuos sólidos separados de acuerdo a la naturaleza del material encontrado en los hogares muestreados se presenta la gráfica 1 que contiene la proporción según el tipo de material y el total por separado.

\section{Gráfica 1}

Total de material separado -Proporción por Tipo de Material

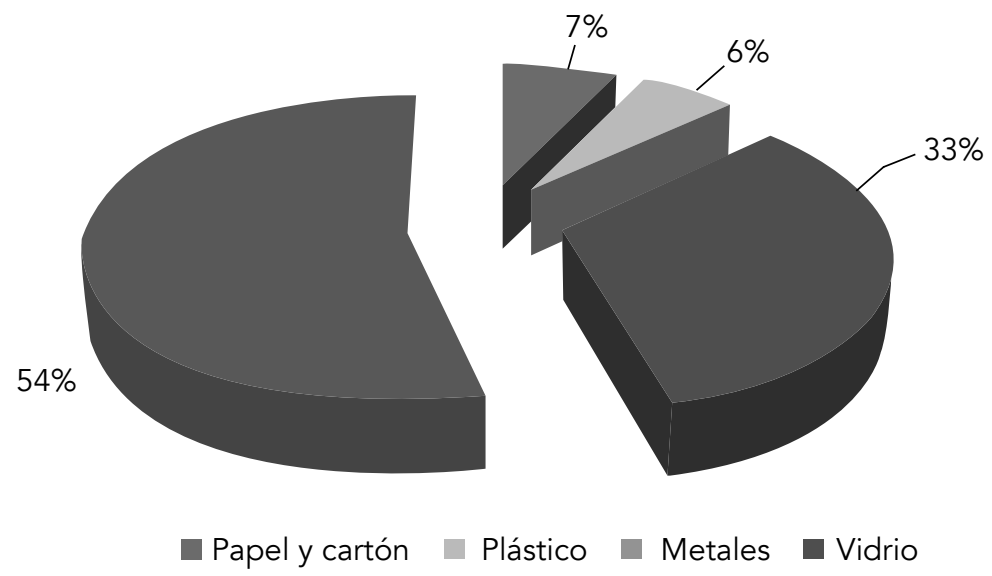

Fuente: Elaboración propia

Si en Cobán se contara con una Gestión Integrada de los Residuos Sólidos, bien acentuada y perfilada de tal manera que se fomentara la cultura ambiental de los habitantes del municipio de Cobán, Alta Verapaz, se podría fomentar, el mejoramiento de las actitudes, valores y comportamiento pro ambientales de la ciuda- danía del lugar, con el valor agregado que de acuerdo a las proporciones de los materiales encontrados en el vertedero municipal se tiene un gran potencial para su comercialización y contar con ingresos económicos. En la gráfica 2 se muestra el total de materiales según la proporción en comercialización: 


\section{Gráfica 2}

Total de Material - Proporción en Comercialización US\$

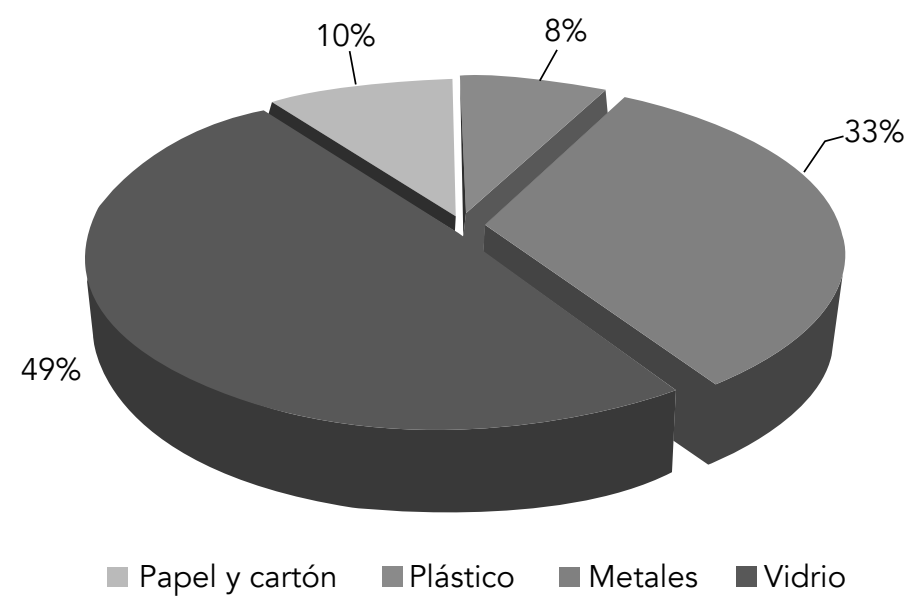

Fuente: Elaboración propia.

Uno de los factores que puede fomentar la GIRS, lo constituye dicha conformación. Si el sector institucional, poblacional y normativo, funcionara de acuerdo a las condiciones requeridas por el municipio, los RS podrían gestionarse de tal manera que puedan comercializarse como material de reutilización y reciclaje, con lo cual se podría dar un valor agregado a la población para incentivarla y poder buscarle otras alternativas al output de RS.

\section{Conclusiones}

Los residuos sólidos no son manejados de manera técnica en la cabecera municipal de Cobán, pues las empresas que los recolectan y transportan no lo hacen clasificando la basura y simplemente los recolectan en las casas y los llevan al depósito ubicado en Sachamach.

Los valores de composición de residuos sólidos municipales o domésticos que se describen en términos de porcentaje en masa, también usualmente en base húmeda y contenidos como materia orgánica, papeles y cartones, escombros, plásticos, textiles, metales, vidrios, huesos, entre otros, tienen un alto potencial de comercialización.
El manejo de los RS en la ciudad de Cobán es llevado a cabo por cuatro empresas recolectoras autorizadas por la municipalidad y prestan el servicio al $60 \%$ de los ciudadanos, con servicio privado costeado por los habitantes en cada vivienda y comercios particulares; sólo el $10 \%$ de los RS los presta la municipalidad con barrido de calles y recolección en comercios y mercados municipales.

El 30 \% de los habitantes de la ciudad de Cobán no contratan los servicios de recolección de RS, prefieren incinerarlos, enterrarlos, tirarlos en orillas de calles, fosas naturales y sitios retirados o transportarlos directamente al vertedero.

La contaminación del aire y el agua, ha sido atribuida también a la gestión inapropiada de los residuos sólidos. Aunque la naturaleza tiene la capacidad de diluir, extender, degradar, absorber o, de otra forma reducir el impacto de los residuos no deseados en la atmósfera, en las lluvias fluviales y en la tierra, han existido desequilibrios ecológicos allí donde se ha excedido la capacidad de asimilación natural.

La separación de los componentes de los residuos sólidos, incluyendo papel, cartón, latas 
de aluminio, vidrio y envases de plástico, en el punto de generación es una de las formas más positivas y eficaces para lograr la recuperación y reutilización de materiales.

El almacenamiento de residuos hospitalarios, corresponde a la acción de cumplir con la reglamentación vigente del Ministerio de Salud y Asistencia Social de Guatemala en tanto se procesen para su aprovechamiento, tratamiento o disposición final por los entes responsables.

\section{Referencias bibliográficas}

AMSA (Autoridad para el manejo sustentable de la cuenca del Lago de Amatitlán, Gt). (2004). La cuenca y el Lago de Amatitlán. Guatemala: JADE.

Building Alliances for Policy Change. (1998). Washington, DC: Johns Hopkins University School of Advanced International Studies Program on Social Change and Development. (Lynch).

CALAS. (2007). Escritos sobre Derecho Ambiental. Centro de Acción Legal Ambiental y Social de Guatemala. Guatemala, Centro América.

CEMAT (Centro Mesoamericano de Estudios sobre Tecnología Apropiada) (2004). Análisis integral nacional. Componente Guatemala.

Centro de Adiestramiento de Seguridad y Ecología (C.A.S.E). (2015). Manual de capacitación "curso básico de seguridad y ecología". México: PEMEX exploración y producción región sur.

Careaga, J. A. (1993) Manejo y Reciclaje de los Residuos de Envases y Embalajes. Chile.: Sedesol. Instituto Nacional de Ecología. Serie Monografías No. 4.

Espinoza, H. (2013). Plan de manejo para la parte alta de la sub cuenca del río Jalapa, con énfasis en el análisis de tres componentes: Aguas residuales y Desechos
Sólidos, manejo y conservación del suelo y gestión de riesgo, Jalapa 2012. Investigación Inferencial, Ingeniero Agrónomo. CUNSURORI-USAC

FIPA-USAID (2001). Documento de apoyo para elaboración de política marco de gestión ambiental. Guatemala. MARN.

ICONTEC. (2009). GTC 24: Guía para la separación en la fuente (Gestión ambiental: Residuos sólidos). Guía técnica colombiana (GTC). Bogotá: Instituto Colombiano de Normas Técnicas y Certificación.

IARNA (Instituto de Agricultura, Recursos Naturales y Ambiente) (2010). Perfil Ambiental de Guatemala: Tendencias y reflexiones sobre la gestión ambiental. (2006). Guatemala: Universidad Rafael Landívar, Facultad de Ciencias Ambientales y Agrícolas, IARNA.

(2009a). (Instituto de Agricultura, Recursos Naturales y Ambiente) Perfil ambiental de Guatemala 2008-2009: las señales ambientales críticas y su relación con el desarrollo. Ciudad de Guatemala (Guatemala): Universidad Rafael Landívar, Facultad de Ciencias Ambientales y Agrícolas, IARNA.

(Instituto de Agricultura, Recursos Naturales y Ambiente) (2009b). Gestión ambiental y gobernabilidad local. Ciudad de Guatemala (Guatemala): Universidad Rafael Landívar, Facultad de Ciencias Ambientales y Agrícolas, IARNA e INGEP.

. (Instituto de Agricultura, Recursos Naturales y Ambiente) (2006). Perfil ambiental de Guatemala: tendencias y reflexiones sobre la gestión ambiental. Ciudad de Guatemala (Guatemala): Universidad Rafael Landívar, Facultad de Ciencias Ambientales y Agrícolas, IARNA.

(Instituto de Agricultura, Recursos Naturales y Ambiente) (2004). Perfil am- 
biental de Guatemala: informe sobre el estado del ambiente y bases para su evaluación sistemática. Ciudad de Guatemala (Guatemala): Universidad Rafael Landívar, Facultad de Ciencias Ambientales y Agrícolas, IARNA.

INE (Instituto Nacional de Estadística). (2003). "Censo Nacional Agropecuario". Ciudad de Guatemala (Guatemala): INE-MAGA.

- (Instituto Nacional de Estadística). (2002). “Encuesta nacional sobre condiciones de vida: perfil de la pobreza en Guatemala". Ciudad de Guatemala (Guatemala): INE.

Instituto Geográfico Nacional. (1985). Hojas Cartográficas de la República de Guatemala. Guatemala: IGN.

JICA (1995). Análisis de la gestión de residuos. Guatemala. Ed. JICA. (Agencia de Cooperación de Japón).

Kiely Gerard, (2001) Ingeniería ambiental, Fundamentos, Entornos, Tecnologías y sistemas de gestión. España: Mc Graw Hill

Leff., E. (2004). Racionalidad Ambiental: la reapropiación social de la naturaleza. México: Siglo XXI Editores.

Lind, D. et al., (2008) Estadística aplicada a los negocios y la economía. México. McGraw-Hill/Interamericana Editores, S.A. de C.V.

Lozano., M. et al. (2007). An analysis of the implementation of an environmental management system in a local public administration. País: Journal of Environmental Management.

MARN (Ministerio de Ambiente y Recursos Naturales) (2009) Informe Ambiental del Estado de Guatemala. GEO Guatemala.

- Ministerio de Ambiente y Recursos Naturales (2001). Situación y evaluación de la calidad ambiental en Guatemala: Diagnóstico y propuestas de solución. Guatemala:

(2003). Situación y evaluación de la calidad ambiental en Guatemala. Diagnóstico y propuestas de solución). Guatemala.

. (2004). Situación y evaluación de la calidad ambiental en Guatemala. Diagnóstico y propuestas de solución. Guatemala:

(2005). Situación y evaluación de la calidad ambiental en Guatemala. Diagnóstico y propuestas de solución. Guatemala.

Martínez, J. (2002). Determinación Cualitativa y Cuantitativa de los Niveles de Contaminación por Desechos Sólidos Orgánicos e Inorgánicos y la Aplicación de Aboneras para la Producción de Compost como una Alternativa viable en el Manejo de los Desechos Orgánicos Producidos en la ciudad de Jalapa. Tesis. Ingeniero Agrónomo. CUNSURORI, JALAPA.

Mijangos, N. (2002). Manejo integral de desechos sólidos del complejo de parques del Instituto de Recreación de los Trabajadores, IRTRA. Parque acuático Xocomil, y temático Xetulul. Guatemala. Tesis. Lic. Biología. Universidad del Valle de Guatemala.

Muñoz, H. (1985). Disposición de basuras en localidades de escasos recursos económicos. Tesis Ing. Civil. Guatemala, Gua. Facultad de Ingeniería, Universidad de San Carlos de Guatemala. s.p.

Odum, E. (1986). Fundamentos de la ecología. México: Editorial Interamericana.

Oliva, B. y Pérez, J. (s.f.) La Contaminación del agua y su impacto en la salud de Guatemala. Facultad de Ciencias Químicas y Farmacia, Universidad de San Carlos de Guatemala. Guatemala: Editorial Universitaria. 
ONU (2009). Indicadores de los Objetivos de Desarrollo del Milenio. Organización de Naciones Unidas, Guatemala.

OPS (Organización Panamericana de Salud) (Gua.) (1994). Lineamientos técnicos para la elaboración de planes de aseo urbano. Guatemala, Gua. V.7, p.43.

Orellana, J. (2013). Diseño de planta de compostaje para el manejo de los desechos sólidos del municipio de San Manuel Chaparrón, Jalapa. 2013. Investigación Inferencial, Ingeniero Agrónomo. CUNSURORI-USAC

PAF-MAYA (2002). Informe de Plan de Acción Forestal Maya. Guatemala. Ed. PAF-MAYA.

PNUD (Programa de Naciones Unidas para el Desarrollo). (2009). Informe de desarrollo humano 2008. Guatemala. Editorial Sur.

- (Programa de Naciones Unidas para el Desarrollo). (2007). Informe de desarrollo humano 2007. Guatemala: Editorial Sur.

- (Programa de Naciones Unidas para el Desarrollo). (2005). Informe Nacional de desarrollo humano. Diversidad étnico-cultural: La ciudadania en un estado plural. Guatemala: Edit. Sur, S.A.

Recinos, Y. (2014). Educación ambiental en el área de salud para el manejo de los desechos sólidos hospitalarios; el caso de los
Centros de Atención Permanente -C.A.P.en el departamento de Jalapa, Guatemala. Tesis de Maestría. CUNSURORI-USAC

Reyes, R. (s.f.) Biodiversidad y Amenazas. Cap. 11. Guatemala: CONAP.

Tchobanogloous G., et al. (1998). Gestión integral de residuos sólidos. España, Mc Graw Hill, Volumen I y II.

Van de Kludndert, A. y J. Anschultz. (2001) Integrated sustenaible waste management-the concept. Tools for decisión makers experience from urban waste expertise programe. Series. Ed. Anne Sheinberg. Netherlands.

Vargas, J. (2008). Basura Cero: Una alternativa sustentable. Disponible en http://www. ecoportal.net/content/view/full/55022. consultado (junio 15 del 2008).

Villanueva, M. (2008). Determinación cualitativa y cuantitativa de los desechos sólidos en la ciudad de Monjas, Jalapa. Investigación Inferencial, Ingeniero Agrónomo. CUNSURORI-USAC

Zorrilla, M. (2005). La influencia de los aspectos sociales sobre la alteración ambiental y la restauración ecológica. In Eds. Sánchez, O; et al. Temas sobre restauración ecológica. México: Instituto Nacional de Ecología. 
